\title{
Virtual Coaching for Older Adults at Home using SMART Goal Supported Behavior Change
}

\author{
Andoni Beristain Iraola \\ eHealth and Biomedical Applications \\ Vicomtech Foundation, Basque \\ Research and Technology \\ Alliance (BRTA) \\ Donostia-San Sebastián, Spain \\ aberistain@vicomtech.org \\ Santiago Hors-Fraile \\ Salumedia Tecnologías \\ Seville, Spain \\ santiagohors@salumedia.com
}

\author{
Roberto Álvarez Sánchez \\ eHealth and Biomedical Applications \\ Vicomtech Foundation, Basque \\ Research and Technology \\ Alliance (BRTA) \\ Donostia-San Sebastián, Spain \\ ralvarez@vicomtech.org \\ Panagiotis Bamidis ${ }^{\dagger}$ \\ Medical Physics Laboratory, \\ School of Medicine \\ Aristotle University of Thessaloniki \\ Thessaloniki, Greece \\ pdbamidis@gmail.com
}

\author{
Despoina Petsani \\ Medical Physics Laboratory, \\ School of Medicine \\ Aristotle University of Thessaloniki \\ Thessaloniki, Greece \\ despoinapets@gmail.com
}
Evdokimos Konstantinidis ${ }^{\dagger}$
WITA, France
Medical Physics Laboratory,
School of Medicine
Aristotle University of Thessaloniki
Thessaloniki, Greece
evdokimosk@gmail.com

${ }^{\dagger}$ Equally contributing senior authors

\begin{abstract}
This paper presents a virtual coach for older adults at home to support active and healthy aging, and independent living. It aids users in their behavior change process for improving on cognitive, physical, social interaction and nutrition areas using SMART goals. To achieve an effective behavior change of the user, the coach relies on the I-Change behavioral change model. Using a combination of projectors, cameras, microphones and support sensors, the older adult's home becomes an augmented reality environment, where common objects are used for projection and sensed. Older adults interact with this virtual coach in their home in a natural way using speech and body gestures (including touch in certain objects) on projected user interfaces in common objects at home.
\end{abstract}

\section{CCS CONCEPTS}

- Applied computing $\sim$ Life and medical sciences $\sim$ Health informatics

KEYWORDS: coaching, i-change, SMART goals, artificial intelligence, recommender systems

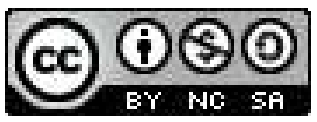

This work is licensed under a Creative Commons Attribution-NonCommercialShareAlike International 4.0 License.

ICMI'20 Companion, October 25-29, 2020, Virtual Event, Netherlands. (c) 2020 Copyright is held by the owner/author(s).

ACM ISBN 978-1-4503-8002-7/20/10.

https://doi.org/10.1145/3395035.3425311

\section{ACM Reference format:}

Andoni Beristain Iraola, Roberto Álvarez Sánchez, Despoina Petsani, Santiago Hors-Fraile, Panagiotis Bamidis \& Evdokimos Konstantinidis. 2020. Virtual Coaching for Older Adults at Home using SMART Goal Supported Behavior Change. In Companion Publication of the 2020 International Conference on Multimodal Interaction (ICMI '20 Companion), October 25-29, 2020, Virtual event, Netherlands. ACM, New York, NY, USA, 5 pages. https://doi.org/10.1145/3395035.3425311

\section{Introduction}

Population life expectancy has increased by 5.5 to 72 years between 2000 and 2016, and healthy life expectancy increased by 4.8 to 63.3 years according to the World Health Organization (WHO) [1]. Despite living longer, natural age-related decline combined with the frequent comorbidity may require that the older adults have some degree of support and supervision. Furthermore, most older adults prefer living on their own instead of staying in a healthcare institution, which does not guarantee better outcomes than at home care [2]. The initiative for active and healthy aging focuses on delaying or even avoiding dependency and being able to enjoy this stage in life.

Coaching is a form of counseling to maximize personal potential, according to the European Commission [3], while the coaching strategy states the plan of actions or guidelines. Optimal guidance is achievable when the coach is aware and adaptable to the user's context, goals and preferences. Siewiorek [4] defined a virtual coach system (VCS) as an always-attentive personalized system that continuously monitors the user's activity and surroundings, and delivers interventions - i.e., intentional messages- when these are desired. Coaching interventions for 
older people to adhere to healthier habits could prolong their independence and leverage costs to the healthcare systems.

According to Turner-Stokes [5], every individual sets lifelong objectives that are considered as goals that last longer to achieve. These long-term goals can be split into smaller medium-term achievements which are consisted of finer grain milestones, the short-term objectives. Finally, those short-term objectives are the ones that can be put into individual actions.

Consequently, goals need to be specific and the progress needs to be measurable and measured. Goals must be achievable for the person and action-oriented, realistic and relevant for the person, and finally time-limited and that is what SMART (specific, measurable, achievable, relevant, and time-limited) acronym stands for. For example, a SMART goal might be [6]: "Being able to walk a quarter of mile to reach my local supermarket within 6 weeks. Tolchin et al [7] proved that SMART goals for treatment planning in psychotherapy were more beneficial than other alternatives.

Effective human coaching for improved health is built upon psychological evidence-based models. Traditional coaching has been understood as a human-to-human relationship. Yet, this concept is shifting as technology becomes part of our lives. The literature review conducted by Wolever et al., 2013 [8] found that only $5 \%$ percent of the analyzed studies about health coaching were only technology based, but this percentage tends to change

In this paper, we present the design of a virtual coach to support older adults maintaining their independence, functional capacity, health status, as well as preserving their physical, cognitive, mental, social well-being and healthy nutritional status, based on radically new ICT approaches combined with a psychological behavioral change model. To describe the virtual coach, first we provide an overview of the system, which supports our virtual coach. Then, we provide the description of the functional elements which constitute our virtual coach, as well as the hardware which supports it. Finally, the conclusions and future work section summarizes the paper and highlights the challenges research avenues.

\section{System overview}

\subsection{Proposed coaching framework}

This work presents the design schema of the CAPTAIN [9] virtual home coach. CAPTAIN's philosophy is to minimize intrusion and avoid wasting older adult's time. Following these principles, CAPTAIN relies on a user interaction based on natural interfaces without wearable devices. Projected augmented reality, micro-projectors, indoor localization devices, voice interaction commands and several services are connected using a distributed and extendable architecture following a publish/subscribe pattern [10] so that modules can cooperate and share data.
During system initialization, the older adult selects and personalizes a set of SMART goals from a catalog organized according to the next categories: nutrition, physical activity, cognitive, or social interaction. Those categories correspond to the four coaching domains supported by the coach. Each of the SMART goals in the catalogue are denoted as SMART goal plugins, or plugins through this paper.

Once the older adult has selected and configured his goals, the coach will trigger the different coaching interventions, based on the plugin contents and its configuration. Periodically the older adult can review the progress of his goals in order to remain motivated and, whenever suited, add, or remove SMART goals, during weekly sessions called coffee sessions. Thus, the older adult has full control about the SMART goals he voluntarily decides to be committed to, being accountable for following or not the coaching recommendations.

\subsection{Multimodal user interface}

The key innovation in the CAPTAIN system user interface was the combination of ubiquitous 3D tangible projections and speech technologies. This project has followed a user centered design. As a result of the first interactions with older adults, 3D tangible projection was not perceived as useful as conventional 2D projection, with body gestures. And speech technologies were perceived as relevant for them. Consequently, we gave more relevance to $2 \mathrm{D}$ projection and speech technologies (i.e. synthesis and recognition). As the user moves from one room to another in his home, the system projects the UI in a conformable location for him (e.g. kitchen fridge) in that room and interacts with him using speech commands (e.g. I want a coffee session, next, one). The user can also interact with arm gestures in tools such as the physical trainer.

\subsection{SMART goal plugin}

A SMART goal plugin is a self-contained set of goals including the actions associated to weekly interventions, trigger rules for the interventions, and configuration parameters so that the older adult can choose the goal difficulty level. In addition to that, it also contains a set of requirements that the user must fulfill to participate. The coach relies completely on these plugins to schedule and dispatch interventions to the user. When an older adult selects and configures a SMART goal plugin, an instance is created with the specific parameters for that goal selected by the user. Each SMART goal plugin contains all the required information to personalize and achieve it, following the SMART concepts [5]. It encompasses two components: the goal management itself, and the goal personalization component.

2.3.1 Goal management. In order to carry out the goal management, the next elements are contained inside the goal management component. First, the set of intervention tools to be used to support the user in achieving the goal. Furthermore, two tightly connected components are also contained: the set of trigger conditions for the interventions (to be delivered by the intervention tools), and the per week intervention plan. 
2.3.2 Goal personalization. The goal personalization information is delivered to the user as a questionnaire during the SMART goal selection and configuration process. The SMART plugin contains an intervention plan per week per combination of parameters. The only mandatory parameter is difficulty. Optional parameters can also be configured depending on the specific SMART goal. For example, some intervention tools can be optional or adjustable, and the goal achievement parameters can be selected based on one's interests.

2.3.3 Intervention tools. The daily intervention tools are an extendable set of software tools that plugins can use to support the behavior change plan with individual interventions, triggered either by scheduling them or under certain user event. Currently, these are the set of available tools:

- Physical Trainer: it is based on the webFitForAll [11] platform for physical exercise and adapts based on user's performance and the analytics about user activity and sedentary activity at home.

- Cognitive Trainer: A specially designed set of cognitive games aiming at training planning and complex behavioral sequences, prospective memory and money management.

- Insight: It provides a piece of knowledge for the user from any of these sources: text presenting patterns automatically inferred from user sensed data or pieces of information related to the SMART plugin.

- Quiz: a short multiple-option answer question game with educational purposes using the spaced learning methodology module of the AdheraHealth platform, adapted to specific SMART goal.

- Recommender: an artificial intelligence selects recommendations relevant to achieve the SMART goal from a pool of potential recommendations in a similar fashion as the system described by Hors-Fraile et al. [12]. These recommendations are grounded in the I-Change behavioral change model [13] using the AdheraHealth platform, which allows personalization based on the information of the older adult profile, and dynamic adaptation to his performance, using his feedback and their activity analytics.

- Reminder list: a static list of reminders configured during system setup for each older adult e.g. for reminding to take you keys before leaving the house.

\section{Functional view}

CAPTAIN's virtual coach approach follows the schema presented in the following figure. It consists of the installation, the weekly (coffee session), the daily agenda and the momentary actions, which are presented below.

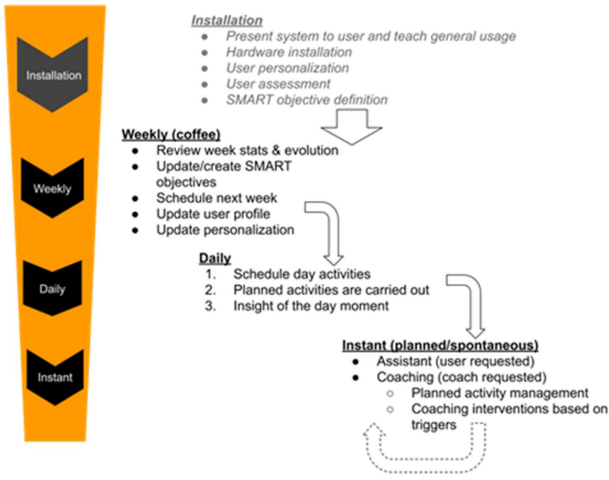

Figure 1: CAPTAIN coaching schema

\subsection{Installation phase}

When users join the CAPTAIN program, a technician and a professional caregiver visit the older adult home to setup the equipment and kickstart the system including: system hardware installation and general configuration, presentation of the system, system personalization, training the user, and the selection of the first SMART objective/s.

The hardware installation and configuration steps require:

- Installing several 3D indoor monitoring sensors (Mentorage $\left.{ }^{\circledR}\right)$, to provide user location and activity sensing.

- Installing several 2D smart projectors, including speakers and microphone, wireless network connectivity and computational power.

- Installing the box device, which provides 3D tangible projection effects, speakers, microphone and camera.

- Calibrating all devices and their connectivity to the home data network.

- Filling general user data for basic personalization like username, home layout plan, system language, avatar type and voice.

The user is also asked to fill a self-reported and private digital questionnaire to collect information about gender, age, height, weight, support requirements, physical state and nutritional habits. To finish the setup phase, the user is presented with the system, including the concept of SMART goals and the user is asked to create one or several SMART goals.

\subsection{SMART goal selection}

To facilitate the older adults in the selection of relevant, specific and measurable goals, he first chooses the coaching area in which he would like to define the goal (i.e. physical, cognitive, social, nutritional), and then fills the interventions in fine grain detail.

Older adults use a catalog to choose from a set of predefined SMART goals (i.e. plugins) with certain configuration parameters, that they can configure, such as the difficulty level. All these goals containing weekly intervention plans for a 
certain period are provided by external institutions. The responsibility of the interventions is shared by the institution providing them and the user deciding to commit to that SMART goal.

By design, the duration of the SMART goal needs to be in the range between 1 and 2 months so that it provides enough time for creating habits, but it is short enough to keep older adults interested and engaged.

Firstly, the coach distributes weekly target interventions per each day, updating the plan for the next day based on the activity on the previous weekdays.

Then, during the day, the coach reminds the older adult about an activity (i.e. intervention provided by an intervention tool) linked to the achievement of a SMART goal based on dynamically managed scheduling and user action detection. The coach asks if the user wants to carry out that intervention. If not, the coach will try to reschedule it for later. Whenever possible, the coach gets a performance feedback of the intervention, otherwise, the coach asks the user. With this feedback, the coach can evaluate the evolution, the preferred activities, as well as habits.

\subsection{Weekly coffee session}

This concept corresponds to a long interaction time called 'coffee session' between the user and the virtual coach. It can be requested by the user at any time, but the system advices periodically the user to have at least one weekly session.

During this coffee session, there is a fixed agenda of topics to be discussed, but the user can skip any of them:

- Review of the last week: It consists of a set of charts obtained after applying data analytics techniques, to review the user's progress and obtain insights. This review includes SMART goals, data provided by the sensors about user behavior as well as prompting motivational messages to the user based on his performance. To increase engagement, the older adult's performance is shown including his historical stats in a supportive and positive way to keep the motivation of the older adult over time.

- Manage SMART goals: the user is asked to check whether he wants to remove any of his active SMART goals or commit to others.

- Coach plan agreement for next week: the coach shows a summary of the interventions proposed for next week considering active SMART goals.

- $\quad$ Update profile data (e.g. weight, preferences).

- Refine the personalization of the coach system (e.g. increase the sound volume of the coach, deactivate notifications at night)

The purpose of this coffee is to foster user engagement with the coaching system, support the behavior change process and provide dynamic system personalization.

\subsection{Day agenda}

The system downloads locally the day plan proposal every day, created using information from the SMART goal plugin and weekly target activity achievement level of previous weekdays. The daily plan includes the intervention services and information for each day.

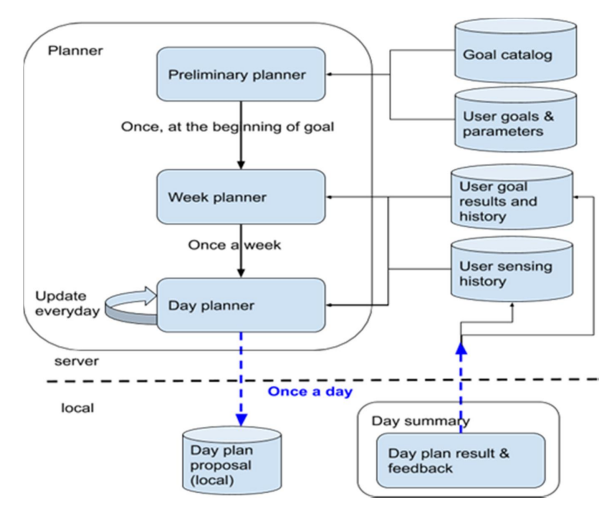

Figure 2: Dynamic day plan based on SMART goal fixed plans

\subsection{Momentary (planned/spontaneous)}

This corresponds to the direct interventions provided directly by the virtual coach. Whenever the virtual coach needs to provide an intervention for the user, it delivers it as a combination of video projections and audio cues. This includes launching the intervention tools (i.e. recipes suggestions), or any other event planned as part of a SMART goal and providing general advice.

\section{Conclusions and future work}

This work presents the design of a virtual coach for older adults. The behavior change recommendations, based on the I-Change model, enable the user to create his own goals and be in the center of the behavior change. Despite the innovative nature of several technologies, many of them relying on artificial intelligence, the main contribution of this system is on blending all that technologies into the older adult's home and unobtrusively monitor and support his everyday life using natural means for user-computer interaction. The system is designed to be easily extendable in order to support the addition of SMART goals in future releases. Additional intervention tools could be also designed and integrated to support the achievement of new SMART goals.

Furthermore, the virtual coach is designed to be unobtrusive in a way that mostly supports the user's intrinsic desire for behavior change. The user is fully empowered by deciding the SMART goals he wants to achieve, supporting him in the steps to achieve them and providing him with tools to monitor his progress. The system follows a structured methodology in order to assist in that direction without intervening with automatic changes. 


\section{REFERENCES}

[1] Patricia S. Abril and Robert Plant, 2007. The patent holder's dilemma: Buy, sell, or troll? Commun. ACM 50, 1 (Jan, 2007), 36-44. DOI: https://doi.org/10.1145/1188913.1188915.

[1] WHO, World health statistics 2019: monitoring health for the SDGs, sustainable development goals. Geneva: World Health Organization; 2019. 2019. ISBN: 978-924-156570-7

[2] L. Boland et al., Impact of home care versus alternative locations of care on elder health outcomes: An overview of systematic reviews, BMC Geriatrics. 2017. DOI: https://doi.org/10.1186/s12877-016-0395-y.

[3] M. Ajdukovic, L. Cajvert, M. Judy, W. Knopf, and H. Kuhn, ECVision. A European Glossary of Supervision and Coaching, no. February, 2015

[4] D. P. Siewiorek, Invited talk: Virtual coaches in health care, 2012 DOI: https://doi.org/10.1109/dsnw.2012.6264659.

[5] L. Turner-Stokes et al., A comprehensive person-centered approach to adult spastic paresis: A consensus-based framework, Eur. 7. Phys. Rehabil. Med., 2018 DOI: https://doi.org/10.23736/S1973-9087.17.04808-0.

[6] J. Bowman, L. Mogensen, E. Marsland, and N. Lannin, The development, content validity and inter-rater reliability of the SMART-Goal Evaluation Method: A standardised method for evaluating clinical goals, Aust. Occup. Ther. 7., 2015. DOI: https://doi.org/10.1111/1440-1630.12218.
[7] B. Tolchin et al. Motivational Interviewing Techniques to Improve Psychotherapy Adherence and Outcomes for Patients With Psychogeni Nonepileptic Seizures, f. Neuropsychiatry Clin. Neurosci., 2019. DOI: https://doi.org/10.1176/appi.neuropsych.19020045.

[8] R. Q. Wolever et al., A Systematic Review of the Literature on Health and Wellness Coaching: Defining a Key Behavioral Intervention in Healthcare, Glob. Adv. Heal. Med., 2013. DOI: https://doi.org/10.7453/gahmj.2013.042.

[9] E. I. Konstantinidis et al., A New Approach for Ageing at Home: The CAPTAIN System, Stud. Health Technol. Inform., vol. 264, pp. 1704-1705, Aug. 2019 DOI: https://doi.org/10.3233/SHTI190606.

[10] K. P. Birman and T. A. Joseph, Exploiting virtual synchrony in distributed systems. In Proceedings of the 11th ACM Symposium on Operating Systems Principles, SOSP 1987. DOI: https://doi.org/10.1145/41457.37515.

[11] E. I. Konstantinidis, A. S. Billis, C. A. Mouzakidis, V. I. Zilidou, P. E. Antoniou, and P. D. Bamidis, Design, implementation, and wide pilot deployment of FitForAll: An Easy to use exergaming platform improving physical fitness and life quality of senior citizens, IEEE 7. Biomed. Heal. Informatics, vol. 20, no. 1, pp 189-200, 2016. DOI: https://doi.org/10.1109/JBHI.2014.2378814.

[12] S. Hors-Fraile et al., Opening the Black Box: Explaining the Process of Basing a Health Recommender System on the I-Change Behavioral Change Model, IEEE Access, 2019. DOI: https://doi.org/10.1109/ACCESS.2019.2957696.

[13] H. de Vries, An Integrated Approach for Understanding Health Behavior; The IChange Model as an Example, Psychol. Behav. Sci. Int. f., 2017. DOI: https://doi.org/10.19080/pbsij.2017.02.555585. 